\title{
Maximum Likelihood Estimators for a Supercritical Branching Diffusion Process
}

\author{
Pablo Olivares ${ }^{1}$ and Janko Hernandez ${ }^{2}$ \\ ${ }^{1}$ Department of Mathematics, Ryerson University, Toronto, ON, Canada M5B 2K3 \\ ${ }^{2}$ School of Business, ITAM, 01000 Mexico City, DF, Mexico \\ Correspondence should be addressed to Pablo Olivares, pablo.olivares@ryerson.ca
}

Received 29 June 2012; Accepted 18 October 2012

Academic Editor: Angelo Plastino

Copyright (c) 2012 P. Olivares and J. Hernandez. This is an open access article distributed under the Creative Commons Attribution License, which permits unrestricted use, distribution, and reproduction in any medium, provided the original work is properly cited.

The log-likelihood of a nonhomogeneous Branching Diffusion Process under several conditions assuring existence and uniqueness of the diffusion part and nonexplosion of the branching process. Expressions for different Fisher information measures are provided. Using the semimartingale structure of the process and its local characteristics, a Girsanov-type result is applied. Finally, an Ornstein-Uhlenbeck process with finite reproduction mean is studied. Simulation results are discussed showing consistency and asymptotic normality.

\section{Introduction}

Some spatial-temporal models are often used to describe the behavior of particles, which are moving randomly in a domain and reproducing after a random time.

We consider a Branching Diffusion Process (BDP), consisting in particles performing independent diffusion movements and having a random numbers of children at random times.

In [1], for example, a simple model of cells with binary splitting after an exponentially distributed random lifetime is considered, where cells move according independent Brownian motions.

More recently, [2] studied a model in order to describe pollution spread through dissemination of particles in the atmosphere. Additionally, the authors take into account the occurrence of particles' mass variations due to random divisions during their lifetimes. For applications in genetic populations see [3]. Also, in [4], the recurrence of a BDP on manifolds is studied. 
In [5], a particle system is considered in a more general context, where interaction among individuals is allowed. There, a link between the associated martingale problem and the infinitesimal generator is established. For a noninteracting BDP, the uniqueness of the martingale problem is found in [6] together with the analysis of the limit behavior of the process.

On the other hand, the statistical approach of this kind of models remains less explored. In [1], under continuous observations upon a fixed time $T$, it obtained the maximum likelihood estimators for the variance and the rate of death of a Brownian motion with a deterministic binary reproduction law. In [7], using a least square approach the parameters of the BDP are also estimated.

In $[8,9]$, a birth and death processes in a flow particle system are considered. There, the absolute continuity of the probability law for the corresponding canonical process is obtained. We follow a similar approach, but allowing the possibility to have more than one particle at birth times, as our case, in which introduces additional complexity due to the exponential growth of the model.

There are many inference results for branching processes as well as for the diffusion process separately; we essentially consider both aspects together via a measure-valued process describing the particle configuration at any time. The functions describing the model (i.e., drift, death rate, and reproduction law) depend on a common unknown parameter.

As in the model mentioned above, technical difficulties arise in writing the corresponding likelihood function. We use a Girsanov theorem for semimartingales, as given, for example, in [10], allowing the passage from a BDP reference measure to another one depending on the true value of the parameter. The semi-martingale structure of the process and its corresponding local characteristics under the change of measure are obtained using Îto's formula.

The covariance matrix of the diffusion part is assumed to be known in order to avoid singularity with respect to the reference measure; otherwise the quadratic variation can be used as a nonparametric estimator of the former.

Expressions for the observed and expected Fisher information measures are provided. In a companion paper, see [11], the asymptotic behavior of these measures is studied, and consequently, the consistency and asymptotic normality of the maximum likelihood estimators.

The organization of the paper is as follows.

In Section 2, we establish the model and the main notations. Also, we give certain sufficient conditions in order to have the existence of diffusion model and the nonexplosion on finite time of the branching part. These conditions are standards in both types of models. In Section 3, we obtain the semi-martingale structure of the model from Îto's formula and we calculate the local characteristics of the BDP. In Section 4, we find the likelihood function of the model using a Girsanov-type theorem for semi-martingales. Finally, in Section 5 we present an example, the Branching Ornstein-Uhlenbeck process, where explicit estimators can be obtained.

\section{Model and Main Notations}

We establish the main features of our model.

Starting from a fixed initial configuration, particles move independently in $\mathbb{R}^{d}$ according to diffusion processes with the same drift and variance. Each particle dies after 
certain random time, depending on its trajectory. At the time of its death, it gives birth to an also random number of particles which continue to move from the ancestor position and reproduce in the same way. $\bigcup_{n=1}^{\infty} \mathbb{N}^{n}$.

Let $\mathcal{U}$ be the set of all particles that can appear in the system; we represent $\mathcal{U}$ by

With every particle $u \in \mathcal{U}$ we associate a random vector $\left(s^{u}, \tau^{u}, N^{u},\left(X_{t}^{u}\right)_{t \in \mathbb{R}_{+}}\right)$where $s^{u}$ and $\tau^{u}$ are its birth and the death times, respectively, taking values on $[0, \infty], X_{t}^{u}$ is its position at time $t$, and $N^{u}$ represents the number of offsprings.

At the initial time $t=0$, we have a configuration given by a finite number of particles denoted by $u_{1}, u_{2}, \ldots, u_{N} \in \mathcal{U}$ at respective deterministic positions $x^{1}, x^{2}, \ldots, x^{N}$. According to notations we establish

$$
s^{u_{1}}=s^{u_{2}}=\cdots=s^{u_{N}}=0, \quad X_{0}^{u_{1}}=x^{1}, \quad X_{0}^{u_{2}}=x^{2}, \ldots, X_{0}^{u_{N}}=x^{N} .
$$

We define recursively the random variables $s^{u}, \tau^{u}$, and $X_{t}^{u}$ in the following way.

Suppose a particle $v$ dies, giving birth to a particle $u$ among its descendants; we set $s^{u}=\tau^{v}$. At time $s^{u}$, the particle $u$ moves according to a diffusion process with drift $b(\cdot)$ and infinitesimal variance $\sigma(\cdot)$ then

$$
X_{t}^{u}=X_{s^{u}}^{u}+\int_{0}^{t} 1_{\left[s^{u}, \infty\right)}(s) b\left(X_{s}^{u}\right) d s+\int_{0}^{t} 1_{\left[s^{u}, \infty\right)}(s) \sigma\left(X_{s}^{u}\right) \cdot d W_{s}^{u} \quad \text { for } t \geq 0,
$$

where $W^{u}=\left(W_{t}^{u}\right)_{t \in R_{+}}$is a standard Brownian motion in $\mathbb{R}^{d}$.

The death rate $\lambda(\cdot)$ function, for a particle located at $x$ at time $t$, satisfies

$$
\operatorname{Pr}\left[\tau^{u} \geq t+\Delta t \mid \tau^{u} \geq t \geq s^{u} ; X_{t}^{u}=x\right]=\lambda(x) \Delta t+o(\Delta t)
$$

Finally, the probability law representing the reproduction law of a particle located at point $x$, and denoted by $\left(p_{k}(x)\right)_{k \in \mathbb{N}}, x \in \mathbb{R}^{d}$ verifies

$$
\operatorname{Pr}\left[N^{u}=k \mid X_{\tau^{u}}^{u}=x\right]=p_{k}(x) .
$$

Processes $\left(W^{u}\right)_{u \in \mathcal{U}}$ and $\left(N^{v}\right)_{v \in \mathcal{U}}$ are independent. where

We describe the process of living particles by the measure-valued process $M=\left(M_{t}\right)_{t \geq 0}$,

$$
M_{t}=\sum_{u \in \mathcal{U}} 1_{\left[s^{u}, \tau^{u}\right)}(t) \delta_{X_{t}^{u}}
$$

Here $\delta_{x}$ denotes the Dirac measure on $\left(\mathbb{R}^{d}, \boldsymbol{B}\left(\mathbb{R}^{d}\right)\right)$, where $\mathbb{B}\left(\mathbb{R}^{d}\right)$ is the Borelian $\sigma$-algebra in $\mathbb{R}^{d}$.

Notice that for $A \in \mathbb{B}\left(\mathbb{R}^{d}\right), M_{t}(A)$ represents the number of living particles in the region $A$ at time $t$. 
The process $M$ is a Markov process called Branching Diffusion Process. For existence and properties see, for example, [5]. This process takes values in

$$
E=\left\{\sum_{i=1}^{n} \delta_{x^{i}}: n=0,1,2, \ldots ; x^{i} \in \mathbb{R}^{d}\right\}
$$

a closed subspace of $\mathbb{M}_{F}\left(\mathbb{R}^{d}\right)$, the space of finite Borel positive measures on $\mathbb{R}^{d}$.

Denote by $C_{b}\left(\mathbb{R}^{d}\right)$ the set of bounded and continuous functions on $\mathbb{R}^{d}$. For every $f \in$ $C_{b}\left(\mathbb{R}^{d}\right)$, we define the norm $\|f\|=\sup \left\{|f(x)|: x \in \mathbb{R}^{d}\right\}$.

For every $\xi \in \mathbb{M}_{F}\left(\mathbb{R}^{d}\right)$ and $f: \mathbb{R}^{d} \rightarrow \mathbb{R}$, measurable we set

$$
\xi(f)=\int_{\mathbb{R}^{d}} f d \xi
$$

We will note for $\langle X, Y\rangle$ and $[X, Y]$ the covariance process and the quadratic covariance process. Also $X^{p}$ is the projection of $X$ in the sense described in [10].

We introduce the following spaces:

$\mho$ : class of right continuous-adapted processes with left limits and with finite variations on finite intervals starting at the origin at time 0 ;

$\mho^{+}$: class of processes in $\mho$ with nondecreasing trajectories;

A: class of processes in $\mho$ with $E \operatorname{Var}(A)_{\infty} \leq \infty$, where $\operatorname{Var}(A)$ is the variation process associated to $A$;

$\mathcal{A}^{+}$: class of processes in $\mho$ with $E A_{\infty} \leq \infty$;

$\mathcal{M}(D)$ : class of uniformly integrable martingales.

Also $\mho_{\mathrm{loc}}, \mho_{\mathrm{loc}^{\prime}}^{+} \mathcal{A}_{\mathrm{loc}^{\prime}}^{+} \mathcal{A}_{\mathrm{loc}}$, and $\mathcal{M}(\mathcal{D})_{\text {loc }}$ are the corresponding local classes.

We take $\left(M_{t}\right)_{t \in R_{+}}$as the canonical process in the stochastic basis $\left(\Omega, \mp, \mathbb{F}, P_{m}\right)$, where $m$ is a given initial configuration, following its usual construction.

By assuming that the functions driving the model depend on an unknown parameter $\theta$, a statistical model associate to the process is considered.

More specifically let $\Theta \subset \mathbb{R}^{m}$ be an open and convex set representing the parametric space and assume that $b, \lambda$, and $p$ depend on a parameter $\theta \in \Theta$, then we have

$$
\begin{gathered}
b: \Theta \times \mathbb{R}^{d} \longrightarrow \mathbb{R}^{d} \quad b(\theta ; x)=b^{\theta}(x)=\left(b_{i}^{\theta}(x)\right)_{i=1, \ldots, d^{\prime}} \\
\sigma: \mathbb{R}^{d} \longrightarrow \mathbb{R}^{d} \otimes \mathbb{R}^{d} \quad \sigma(x)=\left(\sigma_{i j}(x)\right)_{i, j=1, \ldots, d^{\prime}} \\
\lambda: \Theta \times \mathbb{R}^{d} \longrightarrow \mathbb{R}_{+}^{*} \quad \lambda(\theta ; x)=\lambda^{\theta}(x), \\
p: \Theta \times \mathbb{N} \times \mathbb{R}^{d} \longrightarrow[0,1] \quad p(\theta ; k, x)=p_{k}(\theta ; x)=p_{k}^{\theta}(x) .
\end{gathered}
$$

Here $\mathbb{R}^{d} \otimes \mathbb{R}^{d}$ is the space of $d \times d$ real-valued matrices. When no confusion is possible we will note by $|\cdot|$ a norm in the space $\mathbb{R}^{d} \otimes \mathbb{R}^{d}$ as well as the Euclidean norm in $\mathbb{R}^{d}$. These functions define, for a given initial configuration $m$ and any parameter $\theta$, a probability $P_{m}^{\theta}$ in the same way $P_{m}$ is constructed. 
Suppose now these functions satisfy the following properties for every $\theta \in \Theta$.

(A1) Lipshitz Local Condition. For all $n \geq 1$, there exists a constant $C_{n}^{\theta}>0$ such that

$$
\left|b^{\theta}(x)-b^{\theta}(y)\right|+|\sigma(x)-\sigma(y)| \leq C_{n}^{\theta}|x-y| \quad \forall|x| \leq n,|y| \leq n .
$$

(A2) Linear Growth Condition. There exists a constant $K>0$ nondepending on $\theta$ such that

$$
\left|b^{\theta}(x)\right|+|\sigma(x)| \leq K(1+|x|) \quad \forall x \in \mathbb{R}^{d} .
$$

(A3) $\sigma(x)$ is an invertible matrix for all $x \in \mathbb{R}^{d}$, hence

$$
a(x)=\sigma(x)^{t} \sigma(x)
$$

is symmetric and positive definite.

(A4) For all $x \in \mathbb{R}^{d}$ we have

$$
\sum_{k=0}^{\infty} p_{k}^{\theta}(x)=1
$$

(A5) Let

$$
\begin{gathered}
m^{\theta}(x)=\sum_{k=0}^{\infty} k p_{k}^{\theta}(x), \\
\kappa^{\theta}(x)=\sum_{k=0}^{\infty}(k-1)^{2} p_{k}^{\theta}(x)
\end{gathered}
$$

then $\lambda^{\theta}, m^{\theta}$, and $\kappa^{\theta}$ belong to $C_{b}\left(\mathbb{R}^{d}\right)$ with

$$
\lambda^{\theta}(x) \leq\left\|\lambda^{\theta}\right\|, \quad\left|m^{\theta}(x)\right| \leq\left\|m^{\theta}\right\|, \quad\left|\kappa^{\theta}(x)\right| \leq\left\|\kappa^{\theta}\right\| \quad \forall x \in \mathbb{R}^{d} .
$$

(A6) There exist constants $\lambda_{o}^{\theta}>0$ and $m_{o}^{\theta}>1$ such that

$$
\lambda^{\theta}(x) \geq \lambda_{o}^{\theta}, \quad m^{\theta}(x) \geq m_{o}^{\theta}>1 \quad \forall x \in \mathbb{R}^{d} .
$$

Remark 2.1. (A1) and (A2) are standard conditions in order for the existence and uniqueness of the stochastic differential equations describing particle diffusions.

Remark 2.2. The infinitesimal covariance does not depend on $\theta$. In general, we cannot have absolute continuity if $\sigma$ depends on the parameter $\theta$. This seems to be a constrain of the likelihood approach but in some cases it is possible to estimate $\sigma$ using empirical quadratic covariations for example. 
Remark 2.3. The second part of (A6) is a uniform supercritical condition necessary to avoid the almost sure extinction of the branching process.

Let's now define

$$
\underline{r}^{\theta}=\inf _{x \in \mathbb{R}^{d}} \lambda^{\theta}(x)\left(m^{\theta}(x)-1\right), \quad \bar{\gamma}^{\theta}=\sup _{x \in \mathbb{R}^{d}} \lambda^{\theta}(x)\left(m^{\theta}(x)-1\right)
$$

From (A5) and (A6) we have

$$
\begin{gathered}
\bar{r}^{\theta} \leq\left\|\lambda^{\theta}\right\|\left(\left\|m^{\theta}\right\|-1\right), \\
\underline{r}^{\theta} \geq \lambda_{o}^{\theta}\left(m_{o}^{\theta}-1\right)
\end{gathered}
$$

then

$$
0<\underline{r}^{\theta} \leq \lambda^{\theta}(x)\left(m^{\theta}(x)-1\right) \leq \bar{\gamma}^{\theta}<\infty \quad \forall x \in \mathbb{R}^{d}
$$

The expression $\lambda^{\theta}(x)\left(m^{\theta}(x)-1\right)$ is the generalized Malthus parameter, see, for example, $[12,13]$.

We assume that the whole process is observed on an interval $[0, T]$; that is, at every time we observe the entire configuration of particles.

We need to deal with the jumps of the process; to this end we define

$$
\Delta M_{t}=M_{t}-M_{t-}
$$

where $M_{t-}$ is the left limit of process $\left(M_{t}\right)_{t \geq 0}$ at time $t$.

Let's denote by $0<T_{1}<T_{2}<\cdots<T_{n}<\cdots$ the times at which the jumps of the process take place, then, if at time $T_{n}$ a particle dies at position $X_{n}$ and has $K_{n}$ offsprings we have

$$
\Delta M_{T_{n}}=K_{n} \delta_{X_{n}}-\delta_{X_{n}}
$$

The space of jumps is a closed subset of $M_{F}\left(\mathbb{R}^{d}\right)$ defines as

$$
S^{d}=\left\{(k-1) \delta_{x}: k \in \mathbb{N}, x \in \mathbb{R}^{d}\right\}
$$

Let also $\mu^{M}$ be the random measure associated with the jumps of $M$ given by

$$
\mu^{M}(d t, d x)=\sum_{s \leq t} 1_{\left\{\Delta M_{s} \neq 0\right\}} \delta_{\left(s, \Delta M_{s}\right)}
$$


Finally, for every optional function $W$ on $\mathbb{R}_{+} \times S^{d}$ and a random measure $v$ on $\mathcal{B}\left(\mathbb{R}_{+} \times S^{d}\right)$ we define the process $W * v$ by

$$
(W * v)_{t}=\int_{0}^{t} \int_{S^{d}-\{0\}} W(s, x) \mathcal{v}(d s, d x) .
$$

\section{Martingale Representation of the Process and Local Characteristics}

We study now the local characteristics of the process $M$ through the real process $M(f)=$ $\left(M_{t}(f)\right)_{t \geq 0}$.

The following result gives its semi-martingale structure, a useful decomposition of the process in a bounded variation process, a continuous martingale, and a purely discontinuous martingale.

Theorem 3.1. For every function $f: \mathbb{R}^{d} \rightarrow \mathbb{R}$ in $C^{2}\left(\mathbb{R}^{d}\right)$, the process $M(f)$ is decomposed as

$$
M_{t}(f)=M_{0}(f)+\int_{0}^{t} M_{s}\left(A^{\theta} f\right) d s+R f_{t}+i d_{f} * \mu^{M}{ }^{\prime}
$$

where

$$
R f_{t}=\sum_{u \in \mathcal{U}} \int_{0}^{t} 1_{\left[s^{u}, \tau^{u}\right)}(s)\left({ }^{t} D f \cdot \sigma\right)\left(X_{s}^{u}\right) \cdot d W_{s}^{\theta, u}
$$

is a square integrable martingale with zero mean under $\left(\Omega, \mathcal{F}, \mathbb{F}, P_{m}^{\theta}\right)$ and

$$
A^{\theta} f(x)=\frac{1}{2} \sum_{i, j=1}^{d} a_{i j}(x) D_{i, j} f(x)+\sum_{i=1}^{d} b_{i}^{\theta}(x) D_{i} f(x)
$$

is the infinitesimal generator of the common diffusion law followed by the particles and $i d_{f}:(t,(k-$ 1) $\left.\delta_{x}\right) \mapsto(k-1) f(x)$ is optional on $\mathbb{R}_{+} \times S^{d}$.

Here $D_{i}$ and $D_{i, j}$ represent the first derivative with respect to $x_{i}$ and the mixed second derivative with respect to $x_{i}$ and $x_{j}$, respectively, whereas $D={ }^{t}\left(D_{1}, D_{2}, \ldots, D_{d}\right)$.

Proof. We apply Itto's formula to process (2.2) for $f \in C^{2}\left(\mathbb{R}^{d}\right)$. Then we replace $\mathrm{t}$ by $\tau^{u} \wedge t$ and we get

$$
f\left(X_{\tau^{u} \wedge t}^{u}\right)-f\left(X_{s^{u}}^{u}\right)=\int_{0}^{t} 1_{\left[s^{u}, \tau^{u}\right)}(s) A^{\theta} f\left(X_{s}^{u}\right) d s+\int_{0}^{t} 1_{\left[s^{u}, \tau^{u}\right)}(s)\left\{{ }^{t} D f \cdot \sigma\right\}\left(X_{s}^{u}\right) \cdot d W_{s}^{u} .
$$


Adding (3.4) for every $u \in \mathcal{U}$, the right hand side is

$$
\begin{gathered}
\int_{0}^{t}\left\{\sum_{u \in \mathcal{U}} 1_{\left[s^{u}, \tau^{u}\right)}(s) A^{\theta} f\left(X_{s}^{u}\right)\right\} d s+\sum_{u \in \mathcal{U}} \int_{0}^{t} 1_{\left[s^{u}, \tau^{u}\right)}(s)\left\{{ }^{t} D f \cdot \sigma\right\}\left(X_{s}^{u}\right) \cdot d W_{s}^{u} \\
=\int_{0}^{t} M_{s}(A f) d s+R f_{t} .
\end{gathered}
$$

On the other hand, the left hand side can be written as

$$
\begin{aligned}
\sum_{u \in \mathcal{U}: s^{u} \leq t}\left\{f\left(X_{\tau^{u} \wedge t}^{u}\right)-f\left(X_{s^{u}}^{u}\right)\right\} & =\sum_{u \in \mathcal{U}: s^{u} \leq t<\tau^{u}} f\left(X_{t}^{u}\right)+\sum_{u \in \mathcal{U}: \tau^{u} \leq t} f\left(X_{\tau^{u}}^{u}\right)-\sum_{u \in \mathcal{U}: s^{u} \leq t} f\left(X_{s^{u}}^{u}\right) \\
& =M f_{t}-M f_{0}-\sum_{0<s \leq t} \Delta M f_{s} \\
& =M f_{t}-M f_{0}-i d_{f} * \mu^{M}{ }_{t} .
\end{aligned}
$$

By definition $i d_{f} *\left(\mu^{M}-v^{\theta}\right)$ is a local martingale, where $v^{\theta}$ is the compensator of the process $M$ then by adding and subtracting $i d_{f} * v^{\theta}$ we have the following.

Corollary 3.2. For every $f \in C^{2}\left(\mathbb{R}^{d}\right)$ the process

$$
M_{t}(f)-M_{0}(f)-\int_{0}^{t} M_{s}\left(A^{*, \theta} f\right) d s
$$

is, under $\left(\Omega, \mathcal{F}, \mathbb{F}, P_{m}^{\theta}\right)$, a square integrable local martingale with zero mean and quadratic characteristic:

$$
\int_{0}^{t} M_{s}\left({ }^{t} D f \cdot a \cdot D f+\lambda^{\theta} \kappa^{\theta} f^{2}\right) d s
$$

Here,

$$
A^{*, \theta} f=A^{\theta} f+\lambda^{\theta}\left(m^{\theta}-1\right) f
$$

Now, we calculate the local characteristics of the process (2.5). We use the following result which is essentially a particular case of [10, Theorem II.2.42] (see also [14]). 
Proposition 3.3. Let $X$ be a real-adapted process, $h$ a truncating function, $A \in \mathcal{U}$ continuous, $C \in \mho^{+}$ continuous, and $v$ a random measure in $\mathbb{R}_{+} \times \mathbb{R}^{d}$ such that $v(d t, d y)=K_{t}(d y) d t$. Let $B=A+h * v$. Then $X$ is a semimartingale with local characteristics $(B, C, v)$ with respect to a truncating function $h$ if and only if for every $F \in C^{2}(\mathbb{R})$ the process

$$
F\left(X_{t}\right)-F\left(X_{0}\right)-\int_{0}^{t} F^{\prime}\left(X_{s^{-}}\right) d A_{s}-\frac{1}{2} \int_{0}^{t} F^{\prime \prime}\left(X_{s^{-}}\right) d C_{s}-\left\{F\left(X_{s^{-}}+y\right)-F\left(X_{s^{-}}\right)\right\} * v_{t}
$$

is a local martingale.

Proof. It is enough to see that $B \in \mathcal{V}$ is a continuous process and

$$
\begin{aligned}
\int_{0}^{t} F\left(X_{s^{-}}\right) d(h * v)_{s} & =\int_{0}^{t} F\left(X_{s^{-}}\right)\left\{\int_{\mathbb{R}} h(y) K_{s}(d y)\right\} d s \\
& =\int_{(0, t] \times \mathbb{R}} F\left(X_{s^{-}}\right) h(y) v(d s, d y)=\left\{F\left(X_{s^{-}}\right) h(y)\right\} * v_{t}
\end{aligned}
$$

Also,

$$
\left(y^{2} \wedge 1\right) * v_{t}=\int_{0}^{t}\left\{\int_{\mathbb{R}}\left(y^{2} \wedge 1\right) K_{s}(d y)\right\} d s=\int_{0}^{t} H_{s} d s
$$

where $H$ is a nonnegative process then $\left(y^{2} \wedge 1\right) * v \in \mho^{+}$. Moreover, it is continuous therefore predictable and it belongs to $\mathcal{A}_{\text {loc }}^{+} \subset \mathcal{A}_{\text {loc }}$.

We have the following result.

Theorem 3.4. For any $\theta \in \Theta$ and $m \in E^{d}$ there exist a probability $P_{m}^{\theta}$ on as stochastic basis $(\Omega, F, \mathbb{F})$ such that $\left(\Omega, \mathscr{L}, \mathbb{H}, P_{\theta, m}\right)$. We have $M_{0}=m$ a.s. and $\left(B f, C f, v^{f}\right)$ which are the local characteristics of $M(f)$ with respect to $h$ for any $f \in C_{b}^{2}\left(\mathcal{R}^{d}\right)$. The restriction $P_{\theta, m}$ to $\mathcal{F}$ is the only probability in the filtered space $(\Omega, \mathcal{F}, \mathbb{F})$ with these local characteristics. Here $\left(B f, C f, v^{f}\right)$ are given, for any truncating function $h$ by

$$
\begin{gathered}
B f_{t}=\int_{0}^{t} M_{s^{-}}\left(A^{\theta} f\right)(\cdot) d s+h * v_{t}^{f}, \\
C f_{t}=\int_{0}^{t} M_{s^{-}}{ }^{t}(D f(\cdot) a(\cdot) D f(\cdot)) d s
\end{gathered}
$$

and $v^{f}$ on $\mathbb{R}_{+} \times \mathbb{R}^{d}$ as

$$
v^{f}(d t, d y)=M_{t-}\left(\lambda(\cdot) \sum_{k=0}^{\infty} p_{k}^{\theta}(\cdot) \delta_{(k-1) f(\cdot)}(d y)\right) d t
$$


or equivalently, for every optional function $w$ on $\mathbb{R}_{+} \times \mathbb{R}^{d}$ :

$$
w * v_{t}^{f}=\int_{0}^{t} M_{s-}\left(\lambda(\cdot) \sum_{k=0}^{\infty} p_{k}(\cdot) w(s,(k-1) f(\cdot))\right) d s
$$

Proof. From [5, Theorem 3.1], or [6, Chapter 5], we have the existence of a probability measure in $(\Omega, \mathcal{F}, \mathbb{F})$ making (2.5) a BDP with infinitesimal generator $\mathcal{G}^{\theta}=\mathcal{A}^{\theta}+\mathbb{B}^{\theta}$ where

$$
\begin{gathered}
A^{\theta} F(\mu(f))=F^{\prime}(\mu(f)) \mu\left(A^{\theta} f\right)+\frac{1}{2} F^{\prime \prime}(\mu(f)) \mu\left({ }^{t} D f a D f\right), \\
B^{\theta} F(\mu(f))=\mu\left(\lambda^{\theta}(\cdot) \sum_{k=0}^{\infty} p_{k}^{\theta}(\cdot)[F(\mu(f)+(k-1) \delta .(f))-F(\mu(f))]\right) .
\end{gathered}
$$

Moreover, for every non negative function $F \in C_{b}^{2}(\mathbb{R})$ and $f \in C_{b}^{2}\left(\mathbb{R}^{d}\right)$ we have that

$$
F\left(M_{t}(f)\right)-F(m(f))-\int_{0}^{t} \mathcal{G}^{\theta} F\left(M_{s-} f\right) d s
$$

is a local martingale with respect to $\left(\Omega, \mathcal{F}, \mathbb{F}, P_{m}^{\theta}\right)$.

We can write (3.17) as

$$
\begin{aligned}
F( & \left.M_{t}(f)\right)-F\left(M_{0}(f)\right)-\int_{0}^{t} F^{\prime}\left(M_{s-}(f)\right) M_{s-}\left(A^{\theta} f\right) d s-\frac{1}{2} \int_{0}^{t} F^{\prime \prime}\left(M_{s^{-}}\right) M_{s-}\left({ }^{t} D f \cdot a \cdot D f\right) d s \\
& -\int_{0}^{t} M_{s-}\left(\lambda^{\theta} \sum_{k=0}^{\infty} p_{k}^{\theta}\left[F_{f}\left(M_{s-}+(k-1) \delta .\right)-F_{f}\left(M_{s^{-}}\right)\right]\right) d s \\
= & F\left(M_{t} f\right)-F\left(M_{0} f\right)-\int_{0}^{t} F^{\prime}\left(M_{s-} f\right) d\left\{\int_{0}^{s} M_{r-}\left(A^{\theta} f\right) d r\right\} \\
& -\frac{1}{2} \int_{0}^{t} F^{\prime \prime}\left(M_{s-} f\right) d\left\{\int_{0}^{s} M_{r-}\left({ }^{t} D f \cdot a \cdot D f\right) d r\right\} \\
& -\left\{F\left(M_{s-} f+y\right)-F\left(M_{s-} f\right)\right\} * v^{f}(d s, d y)_{t} .
\end{aligned}
$$

From the last expression we apply the precedent proposition and identify the local characteristics as those in expressions (3.13) and (3.15).

\section{Absolutely Continuous Measure Changes, Likelihood Function, and Fisher Information Measures}

In this section, we calculate the likelihood function of the process $M_{t}$ based on a Girsanov theorem for semi-martingales. 
As reference measure we take the one determined by

$$
\begin{gathered}
b^{0}(x)=0, \\
\lambda^{0}(x)=1, \\
p_{k}^{0}(x)=\frac{1}{2^{k+1}}
\end{gathered}
$$

that is, particles moving according to independent Brownian motions without drifts. In the sequel, as we start from a fix deterministic configuration $M_{0}=m$, we will drop the dependence on $m$, then we denote by $P_{0}$ and $P_{\theta}$ the respective probabilities generated by the reference measure and the functions given in (2.8) according to Theorem 3.4. We will denote by $E_{0}$ and $E_{\theta}$ the expectations under $P_{0}$ and $P_{\theta}$, respectively.

It is well known that the semi-martingale structure persists after an absolutely continuous change of the probability measure. In order to see how the local characteristics change with it we construct a probability measure $Q^{\theta}$, absolutely continuous with respect to $P_{0}$, with the same local characteristics than $P_{\theta}$, therefore $Q^{\theta}$ and $P_{\theta}$ are a.s. equal.

Let $\left(B_{0}, C_{0}, v_{0}\right)$ be the local characteristics of the process under $P_{0}$ given by

$$
\begin{gathered}
B_{0} f_{t}=\int_{0}^{t} M_{s-}\left(A_{0} f\right) d s+\left(h_{f} * v_{0}\right)_{t^{\prime}} \\
C_{0} f_{t}=\int_{0}^{t} M_{s-}\left({ }^{t} D f a D f\right) d s, \\
\left(W * v_{0}\right)_{t}=\int_{0}^{t} M_{s-}\left(\lambda^{0}(\cdot) \sum_{k=0}^{\infty} p_{k}^{0}(\cdot) W(s,(k-1) \delta .)\right) d s,
\end{gathered}
$$

where

$$
A_{0} f(x)=\frac{1}{2} \sum_{i, j=1}^{d} D_{i j} f(x) a^{i j}(x)
$$

Equations (3.13) and (3.15) can be, respectively, rewritten as

$$
\begin{gathered}
B f_{t}=\int_{0}^{t} M_{s-}\left(A^{\theta} f\right) d s+h_{f} * v_{t}, \\
C f_{t}=C_{0}, \\
W * v_{t}=\int_{0}^{t} M_{s-}\left(\lambda^{\theta} \sum_{k=0}^{\infty} p_{k}^{\theta} W(s,(k-1) \delta .)\right) d s,
\end{gathered}
$$

where

$$
A^{\theta} f=A_{0} f+{ }^{t} D f \cdot b .
$$

Here $\delta$. refers to the function $x \rightarrow \delta_{x}$. 
Next, we define the function $y: S^{d} \rightarrow \mathbb{R}_{+}$as

$$
y\left((k-1) \delta_{x}\right)=\frac{\lambda(x) p_{k}^{\theta}(x)}{\lambda^{0}(x) p_{k}^{0}(x)}
$$

whenever $\lambda^{0}(x) p_{k}^{0}(x) \neq 0$ and zero are otherwise.

Also we define the following processes on $(\Omega, \mathbb{F}, \mathbb{F})$ :

$$
\begin{gathered}
Y_{t}=\sum_{u \in \mathcal{U}} \int_{0}^{t} 1_{\left[s^{u}, \tau^{u}\right)}\left({ }^{t} b \cdot a^{-1}\right)\left(X_{s}^{u}\right) \cdot d X_{s}^{u}+(y-1) *\left(\mu^{M}-v_{0}\right)_{t^{\prime}} \\
Z_{t}=\exp \left(Y_{t}-\frac{1}{2}\left\langle Y^{c}\right\rangle_{t}\right) \prod_{0<s \leq t}\left(1+\Delta Y_{s}\right) e^{-\Delta Y_{s}} .
\end{gathered}
$$

Note that $Y$ is well defined on the basis $\left(\Omega, \mathcal{F}, \mathbb{F}, P_{0}\right)$. Indeed,

$$
\begin{aligned}
\left\{|y-1| * v_{0}\right\}_{t} & =\int_{0}^{t} M_{s-}\left(\lambda^{0} \sum_{k=0}^{\infty} p^{0}\left|\frac{\lambda^{\theta} p_{k}^{\theta}}{\lambda^{0} p_{k}^{0}}-1\right|\right) d s \\
& \leq \int_{0}^{t} M_{s-}\left(\lambda^{0} \sum_{k=0}^{\infty}\left(\frac{\lambda^{\theta} p_{k}^{\theta}}{\lambda^{0}}+p_{k}^{0}\right)\right) d s \\
& =\int_{0}^{t} M_{s-}\left(\lambda^{0}+\lambda^{\theta}\right) d s \leq\left\|\lambda^{0}+\lambda^{\theta}\right\| \int_{0}^{t} M_{s}(1) d s
\end{aligned}
$$

then $|y-1| * \mathcal{v}_{0} \in \mathcal{A}_{\mathrm{loc}}^{+}\left(P_{0}\right)$ and it is predictable.

Moreover,

$$
E_{0}\left(\left\{|y-1| * v_{0}\right\}_{t}\right) \leq\left\|\lambda^{0}+\lambda\right\| E\left(\int_{0}^{t} M_{s}(1) d s\right)<\infty .
$$

Then $(y-1) *\left(\mu^{M}-v_{0}\right)$ is a purely discontinuous local martingale on $\left(\Omega, \mathcal{F}, \mathbb{F}, P_{0}\right)$.

The first term in $Y$ is a local continuous martingale so the process $Y$ is a local martingale. Their jumps have the form

$$
\Delta Y_{t}=1_{\left[\Delta M_{t} \neq 0\right]}\left(y\left(\Delta M_{t}\right)-1\right)
$$

then

$$
1+\Delta Y_{t}= \begin{cases}1 & \text { if } \Delta M_{t}=0 \\ y\left(\Delta M_{t}\right) & \text { if } \Delta M_{t} \neq 0\end{cases}
$$

Hence, $Z$, the Doleans-Dole exponential local martingale of $Y$, is a local martingale on the same basis. Also $Z \geq 0 P_{0}$-a.s. and $E_{0} Z_{0}=1$. 
Let $\left(R_{n}\right)_{n \in \mathbb{N}}$ be now a sequence of local stopping times for $Z$; we note by $P_{0, R_{n}}$ the restriction of $P_{0}$ to the $\sigma$-algebra $\mathbb{F}_{R_{n}}$ and we define on it the probability measure $Q_{n}$ as $d Q_{n}=$ $Z^{R_{n}} d P_{0, R_{n}}$, where $Z^{R_{n}}$ is the process $Z$ stopped at time $T_{n}$.

We have the following result.

Proposition 4.1. The local characteristics of $Q_{n}$ are given by (3.13) and (3.15).

Proof. Let's note by $\left(B^{n}, C^{n}, v^{n}\right)$ the local characteristics of $M$ under the measure $Q_{n}$.

First, note that if $W$ in $\mathbb{R}_{+} \times S^{d}$ is an optional process then

$$
\begin{aligned}
W *\left(y v_{0}\right)_{t}(\omega)_{t}=\left(W y * v_{0}\right)_{t}(w)_{t} & =\int_{0}^{t} M_{s-}\left(\lambda^{0} \sum_{k=0}^{\infty} p_{k}^{0} W(s,(k-1) \delta .) \frac{\lambda^{\theta} p_{k}^{\theta}}{\lambda^{0} p_{k}^{0}}\right) d s \\
& =\int_{0}^{t} M_{s-}\left(\lambda^{\theta} \sum_{k=0}^{\infty} p_{k}^{\theta} W(s,(k-1) \delta .)\right) d s=W * v_{t},
\end{aligned}
$$

hence $d v=y d v_{0}$ and in a similar way we have $d v^{R_{n}}=y d v_{0}^{R_{n}}$.

On the other hand,

$$
Z_{t}=\exp \left(Y_{t-}-\frac{1}{2}\left\langle Y^{c}\right\rangle_{t}\right)\left(\prod_{0<s<t}\left(1+\Delta Y_{s}\right) e^{-\Delta Y_{s}}\right)\left(1+\Delta Y_{t}\right)
$$

then $Z=Z_{-}(1+\Delta Y)$ and

$$
1_{\left[\Delta M_{t}^{R_{n}} \neq 0\right]} Z_{t}^{R_{n}}=1_{\left[\Delta M_{t}^{R_{n}} \neq 0\right]} Z_{t-}^{R_{n}} y\left(\Delta M_{t}^{R_{n}}\right) .
$$

Thus, we have that for every $D \otimes \mathcal{S}$-measurable function $U: \Omega \times \mathbb{R}_{+} \times S \rightarrow \mathbb{R}_{+}^{d}$,

$$
\sum_{t} 1_{\left[\Delta M_{t} \neq 0\right]} Z_{t} U\left(t, \Delta M_{t}\right)=\sum_{t} 1_{\left[\Delta M_{t} \neq 0\right]} y\left(\Delta M_{t}\right) Z_{t-} U\left(t, \Delta M_{t}\right)
$$

or equivalently

$$
Z U * \mu_{\infty}^{X}=y Z_{-} U * \mu_{\infty}^{X} .
$$

Hence,

$$
E\left(Z U * \mu_{\infty}^{X}\right)=E\left(y Z_{-} U * \mu_{\infty}^{X}\right)
$$

According to [10, Theorem III.3.17], $y Z_{-}$is a version of the conditional expectation $\mathcal{M}_{\mu^{M}}^{P}(Z \mid$ $D \otimes \mathcal{S})$ and consequently $y v_{0}$ is a version of the compensator of $\mu^{X}$ on the basis $\left(\Omega, \mathcal{F}, \mathbb{F}, Q^{\theta}\right)$. Then we have $v^{n}=v$.

Next, we define $N=R f+h_{f} *\left(\mu^{M}-v_{0}\right)$ and $N^{(n)}=\left(N-[N, Y]^{p}\right)^{R_{n}}$, where $N^{R_{n}}$ is the process stopped at time $R_{n}$. 
We can see that $N^{(n)} \in \mathcal{M}_{\text {loc }}\left(Q_{n}\right)$. Indeed, $N^{R_{n}} \in \mathcal{M}_{\text {loc }}\left(P_{0}\right)$ and its jumps

$$
\Delta N^{R_{n}}=1_{\left[\Delta M_{f} \neq 0\right]} h_{f}\left(\Delta M_{f}\right) \leq\|h\|
$$

are bounded; hence combining Theorem III.3.11, Lemma III.3.14 in [10] we have that

$$
N^{(n)}=\left(N-[N, Y]^{p}\right)^{R_{n}} \in \mathcal{M}_{\mathrm{loc}}\left(Q_{n}\right) .
$$

Moreover,

$$
[N, Y]=\left\langle R f, Y^{c}\right\rangle+\sum_{0<s \leq t} \Delta N_{s} \Delta Y_{s}=\left\langle R f, Y^{c}\right\rangle+h_{f}(y-1) * \mu^{M}
$$

and then

$$
\begin{aligned}
N-[N, Y]^{p} & =R f+h_{f} *\left(\mu^{M}-v_{0}\right)-\left\langle R f, Y^{c}\right\rangle-h_{f} * v+h_{f} * v_{0} \\
& =R f-\left\langle R f, Y^{c}\right\rangle+h_{f} *\left(\mu^{M}-v\right) .
\end{aligned}
$$

We can write

$$
N^{(n)}=\left(R f-\left\langle R f, Y^{c}\right\rangle\right)^{R_{n}}+h_{f} *\left(\mu^{M}-v\right)^{R_{n}} \in \mathcal{M}_{\mathrm{loc}}\left(Q_{n}\right) .
$$

As

$$
h_{f} *\left(\mu^{M}-v\right)^{R_{n}}=h_{f} *\left(\mu^{M^{R_{n}}}-v^{R_{n}}\right) \in \mathcal{M}_{\mathrm{loc}}\left(Q_{n}\right)
$$

we have

$$
\left(R f-\left\langle R f, Y^{c}\right\rangle\right)^{R_{n}} \in \mathcal{M}_{\mathrm{loc}}\left(Q_{n}\right)
$$

From (3.1),

$$
\begin{aligned}
M_{t}^{R_{n}} f & =M_{0}^{R_{n}} f+\left(\int_{0}^{t} M_{s}\left(A_{0} f\right) d s+R f\right)^{R_{n}}+i d * \mu_{t}^{M^{R_{n}}} f \\
& =M^{R_{n}} f_{0}+\left(\int_{0}^{t} M_{s}\left(A_{0} f\right) d s+\left\langle R f, Y^{c}\right\rangle\right)^{R_{n}}+\left(R f-\left\langle R f, Y^{c}\right\rangle\right)^{R_{n}}+i d * \mu_{t}^{M^{R_{n}}} f
\end{aligned}
$$


with

$$
\begin{aligned}
\left\langle R f, Y^{c}\right\rangle & =\left\langle\sum_{\mathcal{u} \in \mathcal{U}} \int_{0}^{t} 1_{\left[s^{u}, \tau^{u}\right)}(s)\left\{{ }^{t} D f \cdot \sigma\right\}\left(X_{s}^{u}\right) \cdot d W_{s}^{u}, \sum_{u \in \mathcal{U}} \int_{0}^{t} 1_{\left[s^{u}, \infty\right)}(s)^{t}\left(\sigma^{-1} \cdot b^{\theta}\right)\left(X_{s}^{u}\right) \cdot d W_{s}^{u}\right\rangle \\
& =\int_{0}^{t} M_{s}\left({ }^{t} D f \cdot b^{\theta}\right) d s .
\end{aligned}
$$

We identify $B_{n}$ and $C_{n}$ as (3.13) using Proposition 3.3.

From the previous proposition we get the following.

Theorem 4.2. Under conditions $(A 1)-(A 6)$ in the space $(\Omega, \mathcal{F}, \mathbb{F})$ we have for any $\theta \in \Theta$ that $P_{\theta}$ loc $P_{0}$ with density $Z$ given by (4.8).

The log-likelihood is given by

$$
\begin{aligned}
l_{t}(\theta)= & \sum_{u \in \mathcal{U}: s^{u} \leq t} \int_{s^{u}}^{\tau^{u} \wedge t}\left\{\left({ }^{t} b^{\theta} \cdot a^{-1}\right)\left(X_{s}^{u}\right) \cdot d X_{s}^{u}-\left(\frac{1}{2} b^{t} \cdot a^{-1} \cdot b^{\theta}+\lambda^{\theta}\right)\left(X_{s}^{u}\right) d s\right\} \\
& +\sum_{n=1}^{m_{t}} \ln \lambda^{\theta}\left(X_{n}\right)+\sum_{n=1}^{m_{t}} \ln p_{N_{n}}^{\theta}\left(X_{n}\right),
\end{aligned}
$$

where $m_{t}$ is the number of jumps before time $t$, and $\left(N_{n}-1\right) \delta_{X_{n}}$ is the jump corresponding to time $T_{n}$. Here $P_{\theta} \ll P_{0}$ means that $P_{\theta}$ is locally absolutely continuous with respect to $P_{0}$.

Proof. From Theorem 3.4 we have the existence of the probability measure $P_{\theta}$ with local characteristics given by (3.13) and (3.15); by Proposition 4.1 $P_{\theta}$ and $Q_{n}$ are equal on the $\sigma$ algebra $\mathbb{F}_{T_{n}}$, therefore $P_{T_{n}} \ll\left(P_{0}\right)_{T_{n}}$ with density $Z^{T_{n}}$. By local uniqueness the result can be extended to the $\sigma$-algebra $\mathbb{F}$.

From (4.8) we can write

$$
\begin{aligned}
\ln \left(Z_{t}\right) & =Y_{t}-\frac{1}{2}\left\langle Y^{c}\right\rangle_{t}+\sum_{0<s \leq t} \ln \left(1+\Delta Y_{s}\right)-\sum_{0<s \leq t} \Delta Y_{s} \\
& =Y_{t}^{c}+(y-1) *\left(\mu^{M}-v_{0}\right)_{t}-\frac{1}{2}\left\langle Y^{c}\right\rangle_{t}+\sum_{0<s \leq t} \ln \left(1+\Delta Y_{s}\right)-(y-1) * \mu_{t}^{M} \\
& =Y_{t}^{c}-\left(y * v_{0}\right)_{t}+\left(1 * v_{0}\right)_{t}-\frac{1}{2}\left\langle Y^{c}\right\rangle_{t}+\sum_{0<s \leq t} 1_{\left[\Delta M_{s} \neq 0\right]} y\left(\Delta M_{s}\right)
\end{aligned}
$$


but

$$
\begin{aligned}
\left(y * v_{0}\right)_{t}=(1 & \left.* y v_{0}\right)_{t}=1 * v_{t}=\int_{0}^{t} M_{s-}\left(\lambda^{\theta} \sum_{k=0}^{\infty} p_{k}^{\theta}\right) d s=\sum_{u \in \mathcal{U}} \int_{0}^{t} 1_{\left[s^{u}, \tau^{u}\right)}(s) \lambda^{\theta}\left(X_{s}^{u}\right) d s, \\
\left\langle Y^{c}\right\rangle_{t} & =\sum_{u \in \mathcal{U}} \int_{0}^{t} 1_{\left[s^{u}, \tau^{u}\right)}(s)\left({ }^{t} b^{\theta} \cdot{ }^{t}\left(\sigma^{-1}\right)\right)\left(X_{s}^{u}\right)\left(\sigma^{-1} \cdot b^{\theta}\right)\left(X_{s}^{u}\right) \cdot d\left\langle W^{u}\right\rangle_{s} \\
& =\sum_{u \in \mathcal{U}} \int_{0}^{t} 1_{\left[s^{u}, \tau^{u}\right)}(s)\left({ }^{t} b^{\theta} \cdot a^{-1} \cdot b^{\theta}\right)\left(X_{s}^{u}\right) d s .
\end{aligned}
$$

Then

$$
\begin{aligned}
\ln \left(Z_{t}\right)= & \sum_{u \in \mathcal{U}: s^{u} \leq t} \int_{s^{u}}^{\tau^{u} \wedge t}\left\{\left({ }^{t} b^{\theta} \cdot a^{-1}\right)\left(X_{s}^{u}\right) \cdot d X_{s}^{u}-\left(\frac{1}{2}{ }^{t} b^{\theta} \cdot a^{-1} \cdot b^{\theta}+\lambda^{\theta}\right)\left(X_{s}^{u}\right) d s\right\} \\
& +\sum_{n=1}^{m_{t}}\left\{\ln \lambda^{\theta}\left(X_{n}\right)+\ln p_{N_{n}}^{\theta}\left(X_{n}\right)\right\}-\sum_{n=1}^{m_{t}} \ln \lambda^{0} p_{N_{n}}^{0}\left(X_{n}\right)+\left(1 * \mathcal{v}_{0}\right)_{t} .
\end{aligned}
$$

Neglecting terms nondepending on $\theta$ we get (4.28).

Next, we give expressions for the Fisher information and related measures. For details in the proofs and their asymptotic analysis we refer to [11].

We denote by $\left(\dot{l}_{t}(\theta)\right)_{t \geq 0}$ the score process, where the dot means the gradient with respect to the parameter $\theta$. It is well known that $\left(\dot{l}_{t}(\theta)\right)_{t \geq 0}$ is a zero mean martingale under $P_{\theta}$. Its quadratic variation $J_{t}(\theta)=[\dot{l}(\theta)]_{t}$ is the observed incremental information and the associate variance process $I_{t}(\theta)=\langle\dot{l}(\theta)\rangle_{t}$ is the expected incremental information. We denote by $j_{t}(\theta)=-\ddot{l}_{t}(\theta)$ the Fisher observed information. Finally, the expected information is $i_{t}(\theta)=E_{\theta}\left(\dot{l}_{t}(\theta){ }^{t} i_{t}(\theta)\right)$, see, for example, [15]. Among these four quantities we have the following relation:

$$
i_{t}(\theta)=E_{\theta}\left(J_{t}(\theta)\right)=E_{\theta}\left(I_{t}(\theta)\right)=E_{\theta}\left(j_{t}(\theta)\right) .
$$

We have the following result.

Proposition 4.3. If in addition to (A1)-(A6), we assume the following conditions:

(B1) The function $x \mapsto \sigma^{-1}(x)$ is bounded, that is, $\left|\sigma^{-1}(x)\right| \leq\left\|\sigma^{-1}\right\|<\infty$ for every $x \in \mathbb{R}^{d}$.

(B2) There exist constants $B_{1}, B_{2}, B_{3}, \Lambda_{1}, \Lambda_{2}, P_{1}$, and $P_{2}$, such that for every $\theta \in \Theta$, all $x \in \mathbb{R}^{d}$, all $k \in \mathbb{N}$, and all $i, j, l=1, \ldots, m$, the following inequalities are satisfied:

$$
\begin{gathered}
\left|D_{i} b^{\theta}(x)\right| \leq B_{1}, \quad\left|D_{i j} b^{\theta}(x)\right| \leq B_{2}, \quad\left|D_{i j l} b^{\theta}(x)\right| \leq B_{3}, \\
\left|D_{i} \lambda^{\theta}(x)\right| \leq \Lambda_{1}, \quad\left|D_{i j} \lambda^{\theta}(x)\right| \leq \Lambda_{2}, \\
\left|D_{i} \ln p_{k}^{\theta}(x)\right| \leq P_{1}, \quad\left|D_{i j} \ln p_{k}^{\theta}(x)\right| \leq P_{2},
\end{gathered}
$$


then $J(\theta), I(\theta)$, and $i(\theta)$ are given by,

$$
\begin{gathered}
J_{t}(\theta)_{i j}=\int_{0}^{t} M_{s}\left({ }^{t} D_{i} b^{\theta} a^{-1} D_{j} b^{\theta}\right) d s+\left\{D_{i}\left(\ln \lambda^{\theta}+\ln p_{k}^{\theta}\right) \cdot D_{j}\left(\ln \lambda^{\theta}+\ln p_{k}^{\theta}\right)\right\} * \mu_{t}^{M}, \\
I_{t}(\theta)_{i j}=\int_{0}^{t} M_{s}\left(\xi_{i j}^{\theta}\right) d s, \\
i_{t}(\theta)_{i j}=\sum_{n=0}^{M_{0}(1)} \int_{0}^{t} \mathbb{E}_{x^{n}}^{\theta}\left[\xi_{i j}^{\theta}\left(Y_{s}\right) \exp \left\{\int_{0}^{s} \lambda^{\theta}\left(m^{\theta}-1\right)\left(Y_{r}\right) d r\right\}\right] d s,
\end{gathered}
$$

where

$$
\xi_{i j}^{\theta}={ }^{t} D_{i} b^{\theta} a^{-1} D_{j} b^{\theta}+\lambda^{\theta} \cdot D_{i} \ln \lambda^{\theta} \cdot D_{j} \ln \lambda^{\theta}+\lambda^{\theta} \cdot \sum_{k=0}^{\infty} D_{i} \ln p_{k}^{\theta} \cdot D_{j} \ln p_{k}^{\theta} \cdot p_{k}^{\theta} .
$$

\section{A Branching Ornstein-Uhlenbeck Process}

We consider a BDP where particles move according to an Ornstein-Uhlenbeck process on $\mathbb{R}$, then

$$
X_{t}=\varphi \int_{0}^{t} X_{s} d s+W_{t}
$$

The death rate $\lambda \in(0, \infty)$ does not depend on the position; hence every particle has an exponential distributed lifetime independently of the trajectory.

Its reproduction law $\pi=\left(\pi_{k}\right)_{k \in \mathcal{N}}$ satisfies

$$
\begin{gathered}
\pi_{1}=0, \\
\sum_{k=0}^{\infty} k \pi_{k}<\infty,
\end{gathered}
$$

where $\pi_{k}$ refers to the probability that a particle has $k$ offsprings. Then the parameter is $\theta=$ $(\varphi, \lambda, \pi) \in \Theta$ where $\Theta \subset \mathbb{R} \times(0, \infty) \times[0,1]^{\mathbb{N}}$.

So we write

$$
\begin{gathered}
b^{\theta}(x)=\varphi x, \\
\sigma^{\theta}(x)=1, \\
\lambda^{\theta}(x)=\lambda, \\
p^{\theta}(x)=\pi .
\end{gathered}
$$


From (4.28) we get

$$
\begin{aligned}
l_{t}(\theta)= & \sum_{u \in \mathcal{U}: s^{u} \leq t} \int_{s^{u}}^{\tau^{u} \wedge t}\left\{\varphi X_{s}^{u} d X_{s}^{u}-\frac{1}{2}\left(\varphi X_{s}^{u}\right)^{2} d s-\lambda d s\right\}+m_{t} \ln \lambda+\sum_{n=1}^{m_{t}} \ln \pi_{N_{n}} \\
= & \varphi \sum_{u \in \mathcal{U}: s^{u} \leq t} \int_{s^{u}}^{\tau^{u} \wedge t} X_{s}^{u} d X_{s}^{u}-\frac{\varphi^{2}}{2} \sum_{u \in \mathcal{U}: s^{u} \leq t} \int_{s^{u}}^{\tau^{u} \wedge t}\left(X_{s}^{u}\right)^{2} d s \\
& -\lambda \sum_{u \in \mathcal{U}: s^{u} \leq t}\left(\tau^{u} \wedge t-s^{u}\right)+m_{t} \ln \lambda+\sum_{n=1}^{m_{t}} \ln \pi_{N_{n}} .
\end{aligned}
$$

Noting that

$$
\sum_{u \in \mathcal{U}: s^{u} \leq t}\left(\tau^{u} \wedge t-s^{u}\right)=K_{0} T_{1}+K_{1}\left(T_{2}-T_{1}\right)+\cdots+K_{m_{t}-1}\left(T_{m_{t}}-T_{m_{t}-1}\right)+K_{m_{t}}\left(t-T_{m_{t}}\right)
$$

where $K_{n}$ is the number of particles alive on the interval $\left[T_{n}, T_{n+1}\right)$ then

$$
K_{n}= \begin{cases}N_{0} & \text { if } n=0 \\ N_{0}+\left(N_{1}-1\right)+\cdots+\left(N_{n}-1\right) & \text { if } n>0\end{cases}
$$

where $N_{0}$ is the number of ancestors and

$$
S_{t}=K_{0} T_{1}+K_{1}\left(T_{2}-T_{1}\right)+\cdots+K_{m_{t}-1}\left(T_{m_{t}}-T_{m_{t}-1}\right)+K_{m_{t}}\left(t-T_{m_{t}}\right) .
$$

We finally have

$$
\begin{aligned}
l_{t}(\theta)= & \frac{\varphi}{2}\left(\sum_{u \in \mathcal{U}: s^{u} \leq t}\left\{\left(X_{\tau^{u} \wedge t}^{u}\right)^{2}-\left(X_{s^{u}}^{u}\right)^{2}\right\}-S_{t}\right)-\frac{\varphi^{2}}{2} \sum_{u \in \mathcal{U}: s^{u} \leq t} \int_{s^{u}}^{\tau^{u} \wedge t}\left(X_{s}^{u}\right)^{2} d s \\
& -\lambda S_{t}+m_{t} \ln \mathcal{\lambda}+\sum_{n=1}^{m_{t}} \ln \pi_{N_{n}} .
\end{aligned}
$$

From (5.8) we obtain the maximum likelihood estimators:

$$
\begin{gathered}
\widehat{\varphi}_{t}=\frac{\sum_{u \in \mathcal{U}: s^{u} \leq t}\left\{\left(X_{\tau^{u} \wedge t}^{u}\right)^{2}-\left(X_{s^{u}}^{u}\right)^{2}\right\}-S_{t}}{2 \sum_{u \in \mathcal{U}: s^{u} \leq t} \int_{s^{u}}^{\tau^{u} \wedge t}\left(X_{s}^{u}\right)^{2} d s}, \\
\widehat{\lambda}_{t}=\frac{m_{t}}{S_{t}} \\
\widehat{\pi}_{n, t}=\frac{r_{t}^{n}}{m_{t}} .
\end{gathered}
$$

Here $r_{t}^{n}$ is the number of splitting on $(0, t)$ resulting in $n$ offsprings. 
Table 1: Parameter estimates of an Ornstein-Uhlenbeck process with two or three splitting. Five trajectories are simulated with parameters $\phi=0.1, \lambda=0.05$ and $\pi_{2}=\pi_{3}=1 / 2$.

\begin{tabular}{lccc}
\hline$\widehat{\varphi}$ & $\hat{\imath}$ & $\widehat{\pi_{2}}$ & $\widehat{\pi_{3}}$ \\
\hline 0.10003 & 0.05139 & 0.499 & 0.501 \\
0.10002 & 0.05152 & 0.525 & 0.475 \\
0.10002 & 0.04974 & 0.524 & 0.476 \\
0.10033 & 0.05028 & 0.498 & 0.502 \\
0.10005 & 0.04852 & 0.524 & 0.476 \\
\hline
\end{tabular}

Moreover, we have the following results:

$$
\begin{aligned}
& \hat{\lambda}_{t} \stackrel{\text { a.s. }}{\longrightarrow} \lambda, \\
& \widehat{\pi_{n t}} \stackrel{\text { a.s. }}{\longrightarrow} \pi_{n} \quad \forall n, \\
& \sqrt{m_{t}}\left(\frac{\lambda}{\hat{\lambda}_{t}}-1\right) \stackrel{\varrho}{\longrightarrow} N(0,1),
\end{aligned}
$$

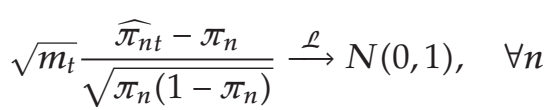

suggesting consistency and asymptotic normality of the estimators in a more general context.

We perform a simulation analysis for the model above in the following way.

Equation (5.1) is discretized as

$$
X_{t+h}=X_{t}+\varphi \int_{t}^{t+h} X_{s} d s+\left(W_{t+h}-W_{t}\right)
$$

For small $h$ we take:

$$
X_{t+h} \approx X_{t}+\varphi h X_{t}+\xi^{h},
$$

where $\xi^{h} \sim N(0, h)$. As initial parameters we take

$$
\begin{gathered}
\varphi=0.1, \\
\lambda=0.05, \\
\pi_{2}=\pi_{3}=\frac{1}{2} .
\end{gathered}
$$

Numerical results from simulated trajectories are shown in Table 1. The particle system is observed until the time of the 1000th reproduction. 


\section{Acknowledgments}

This research has been partially supported by the Natural Sciences and Engineering Research Council of Canada. Also, the authors would like to thank the support received from the Asociación Mexicana de Cultura

\section{References}

[1] S. R. Adke and S. R. Dharmadhikari, "The maximum likelihood estimation of coefficient of diffusion in a birth and diffusion process," Biometrika, vol. 67, no. 3, pp. 571-576, 1980.

[2] L. G. Gorostiza, "A measure valued process arising from a branching particle system with changes of mass," in Measure-Valued Processes, Stochastic Partial Differential Equations, and Interacting Systems, D. A. Dawson, Ed., vol. 5 of CRM Proceedings and Lecture Notes, pp. 111-118, American Mathematical Society, Providence, RI, USA, 1994.

[3] T. Huillet, "A branching di usion model of selection: from the neutral wright-fisher case to the one including mutations," International Mathematical Forum, vol. 7, no. 1, pp. 1-36, 2012.

[4] A. Grigor'yan and M. Kelbert, "Recurrence and transience of branching diffusion processes on Riemannian manifolds," The Annals of Probability, vol. 31, no. 1, pp. 244-284, 2003.

[5] S. Roelly and A. Rouault, "Construction et propriétés de martingales des branchements spatiaux interactifs," International Statistical Review, vol. 58, no. 2, pp. 173-189, 1990.

[6] S. N. Ethier and T. G. Kurtz, Markov Processes, Wiley Series in Probability and Mathematical Statistics: Probability and Mathematical Statistics, John Wiley \& Sons, New York, NY, USA, 1986.

[7] R. Kulperger, "Parametric estimation for simple branching diffusion processes. II," Journal of Multivariate Analysis, vol. 18, no. 2, pp. 225-241, 1986.

[8] M. J. Phelan, "A Girsanov transformation for birth and death on a Brownian flow," Journal of Applied Probability, vol. 33, no. 1, pp. 88-100, 1996.

[9] R. Höpfner and E. Löcherbach, "On local asymptotic normality for birth and death on a flow," Stochastic Processes and their Applications, vol. 83, no. 1, pp. 61-77, 1999.

[10] J. Jacod and A. N. Shiryaev, Limit Theorems for Stochastic Processes, vol. 288 of Grundlehren der Mathematischen Wissenschaften, Springer, Berlin, Germany, 1987.

[11] J. Hernandez, P. Olivares, and M. Escobar, "Asymptotic behavior of maximum likelihood estimators in a branching diffusion model," Statistical Inference for Stochastic Processes, vol. 12, no. 2, pp. 115-137, 2009.

[12] P. Jagers, Branching Processes with Biological Applications, Wiley-Interscience, London, UK, 1975.

[13] S. Asmussen and H. Hering, Branching Processes, vol. 3 of Progress in Probability and Statistics, Birkhäuser, Boston, Mass, USA, 1983.

[14] M. Métivier, Semimartingales, vol. 2 of de Gruyter Studies in Mathematics, Walter de Gruyter, Berlin, Germany, 1982.

[15] B. L. S. Prakasa Rao, Semimartingales and Their Statistical Inference, vol. 83 of Monographs on Statistics and Applied Probability, Chapman \& Hall, Boca Raton, Fla, USA, 1999. 


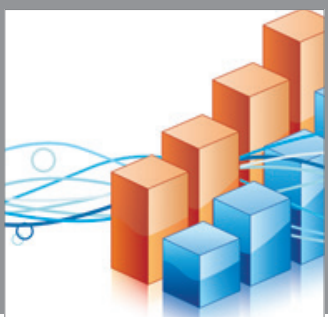

Advances in

Operations Research

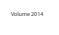

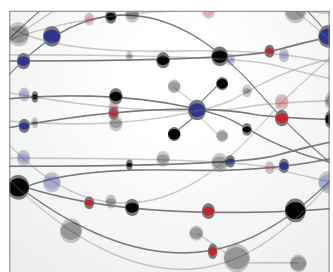

\section{The Scientific} World Journal


International Journal of

Mathematics and

Mathematical

Sciences
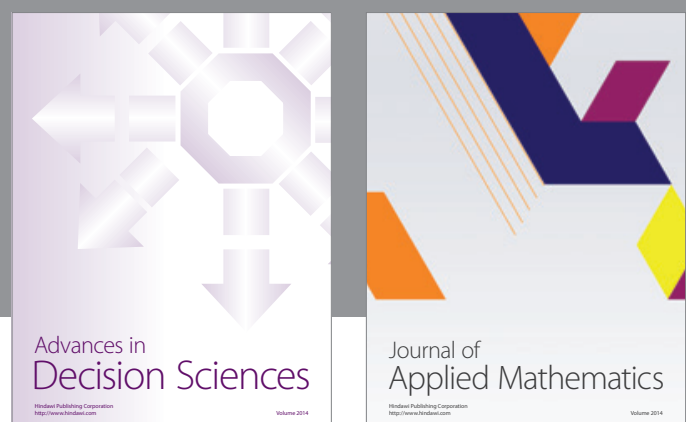

Journal of

Applied Mathematics
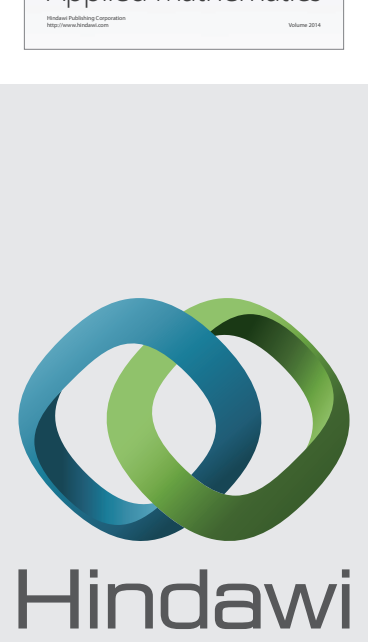

Submit your manuscripts at http://www.hindawi.com
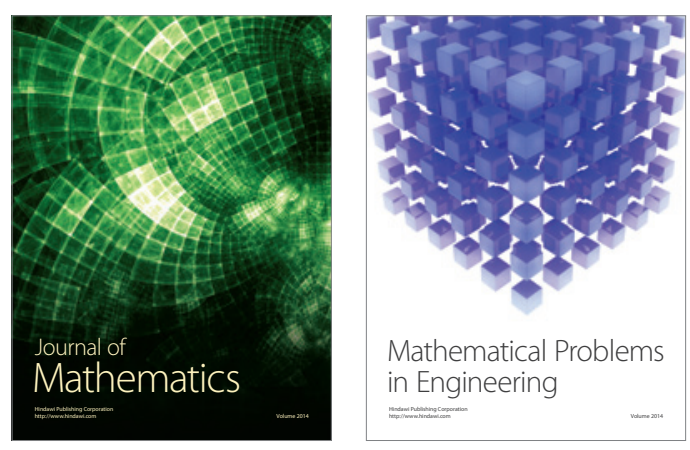

Mathematical Problems in Engineering


Journal of

Function Spaces


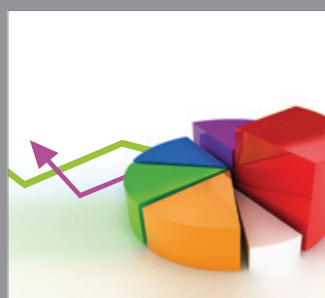

ournal of

Probability and Statistics

Promensencen
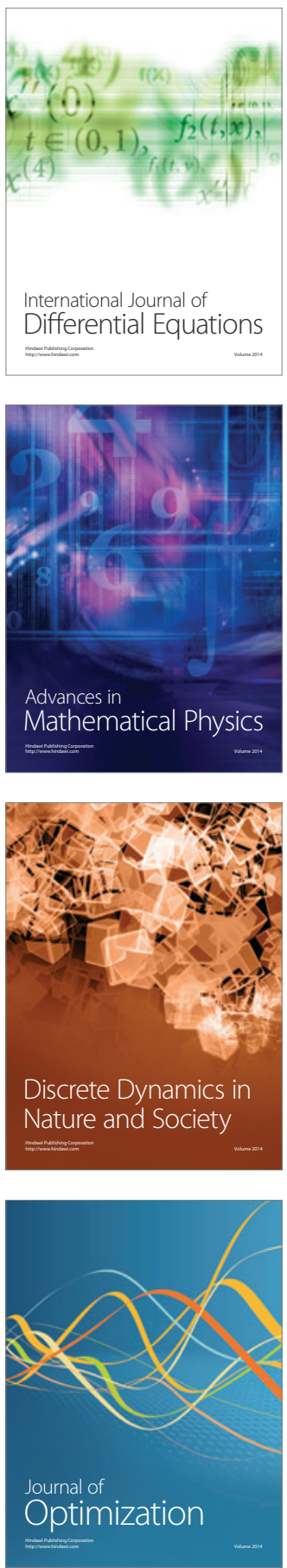